\title{
CRIAÇÃO DE MODELOS ANALÍTICOS DE ESTRUTURAS COM GEOMETRIA COMPLEXA
}

\author{
Luís Ribeirinho $^{(1)}$, Miguel Albergaria ${ }^{(1)}$ \\ (1) TPF - CONSULTORES DE ENGENHARIA E ARQUITETURA, S.A., Lisboa
}

\begin{abstract}
Resumo
Os projetos de Estruturas concebidos na TPF - CONSULTORES DE ENGENHARIA E ARQUITETURA S.A. através da metodologia BIM têm vindo a ser desenvolvidos com recurso ao Revit. Neste programa, a modelação de estruturas com geometria complexa dificilmente poderá ser feita de modo eficiente com recurso a elementos nativos que incorporem, de forma automática, o modelo analítico, o que inviabiliza a interoperabilidade com os programas de cálculo. Para ultrapassar esta limitação desenvolveram-se procedimentos e rotinas que permitem criar os modelos de cálculo, aproveitando a geometria modelada no Revit.

O primeiro passo consiste na exportação da geometria, que é realizada com recurso a uma pequena rotina desenvolvida no Dynamo. A geometria gerada é então importada para o Rhinoceros onde, com auxílio de uma rotina desenvolvida em Grasshopper, é definida a malha de elementos finitos, que é posteriormente utlizada para gerar o modelo em SAP2000 a partir do plug-in da Geometry Gym.

Este processo permite reduzir e simplificar significativamente o trabalho de modelação do modelo analítico.
\end{abstract}

\section{Introdução}

Nas últimas décadas, os computadores penetraram em todos os aspetos do quotidiano da engenharia, transformando profundamente a forma como certas tarefas são executadas. Ao longo desses anos, a relação entre os engenheiros e o software foi sofrendo alterações. Inicialmente, qualquer engenheiro que quisesse usar um computador, teria de escrever o seu próprio código. Mais tarde, começaram a surgir grupos de programadores no seio das empresas, responsáveis por manter o software desenvolvido internamente. À medida que a complexidade do software foi crescendo, emergiu um mercado altamente competitivo de software comercial. Atualmente, a maioria dos engenheiros usa software comercial. Apenas uma pequena franja se dedica a selecionar, adaptar e integrar software nas empresas [1].

Contudo, avizinha-se uma nova mudança deste paradigma com a proliferação dos Citizen Developers, patrocinada pelos ambientes de programação visual. De acordo com a consultora 
Gartner, um Citizen Developers é um utilizador que cria aplicações de negócios para consumo de outros utilizadores, recorrendo a ambientes aprovados pela empresa [2].

No presente artigo, analisa-se uma área particular da engenharia assistida por computadores (Computer-Aided Engineering - CAE), a mecânica computacional, que envolve a aplicação de mecânica, matemática e métodos numéricos na engenharia. Utilizam-se modelos matemáticos para representar fenómenos físicos que ocorrem em sistemas de engenharia. Esses modelos ajudam a simular o comportamento desses sistemas, permitindo verificar o dimensionamento e otimizar as soluções. O método mais utilizado da mecânica computacional é o método dos elementos finitos (Finite Element Method - FEM). Neste método, os objetos são discretizados em malhas de nós e elementos. A análise de elementos finitos requer grandes quantidades de dados de entrada e gera habitualmente muitos dados de saída. Os desafios relacionados com a interação do utilizador são a preparação dos dados de entrada e o pós-processamento dos dados de saída. A geração da malha, que consumia muito tempo, se fosse feita manualmente, acabou por ser automatizada. Atualmente, os programas de análise estrutural permitem gerar as malhas a partir do modelo geométrico [1].

O referido modelo geométrico poderá ser definido no programa de cálculo, ou, preferencialmente, importado, aproveitando a modelação feita no software de modelação, onde a estrutura foi definida, ou, de forma mais rigorosa, aproveitando o modelo analítico dessa estrutura. Um modelo analítico é uma representação tridimensional simplificada do modelo físico estrutural. Para além da geometria, o modelo analítico contém informação sobre as propriedades dos materiais e as cargas a que os elementos estruturais estão sujeitos.

\section{Interoperabilidade Revit-SAP2000}

No caso do Revit [3], o modelo analítico é gerado automaticamente, quando o modelo físico é criado. Pelo menos, para alguns elementos, como pilares, vigas, lajes, paredes ou fundações, desde que tenham geometrias regulares. O problema surge quando precisamos de criar geometrias que não podem ser representadas com recurso às ferramentas base de edição disponíveis para aqueles elementos, como é o caso das paredes de galerias subterrâneas - túneis de secção irregular (Figura 1). Estes elementos têm de ser customizados e não têm modelo analítico associado (Figura 2). 
ptBIM $2020-3^{\circ}$ Congresso Português de Building Information Modelling

26 e 27 de novembro e 4 de dezembro de 2020, Faculdade de Engenharia da Universidade do Porto

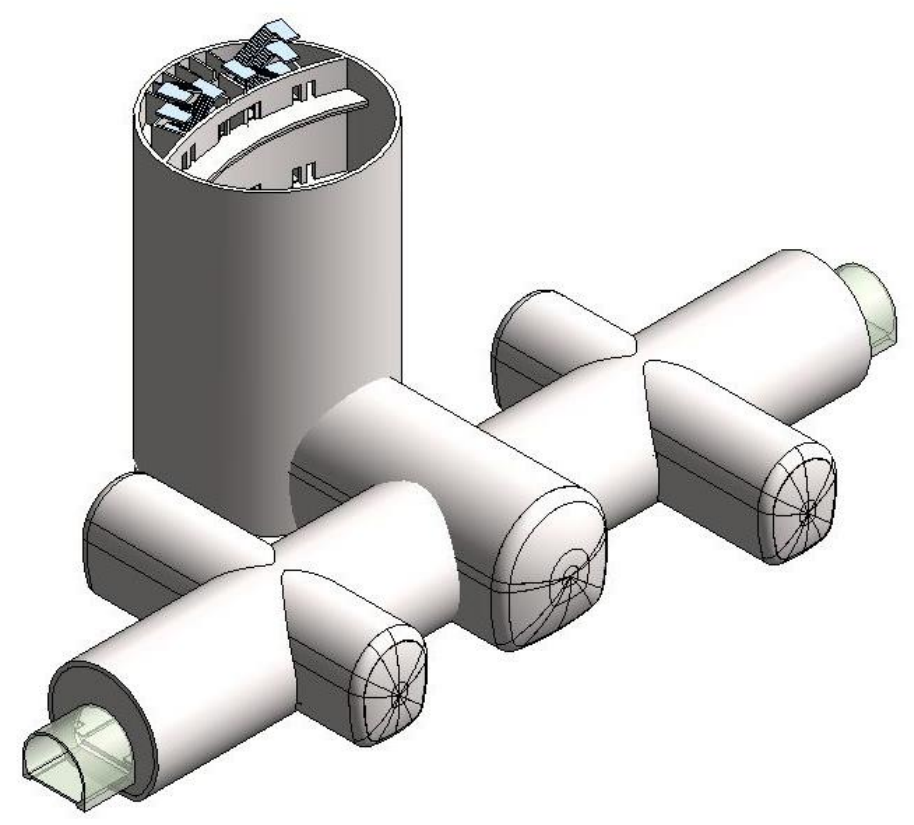

Figura 1: Modelo geométrico da estrutura no Revit 2019.

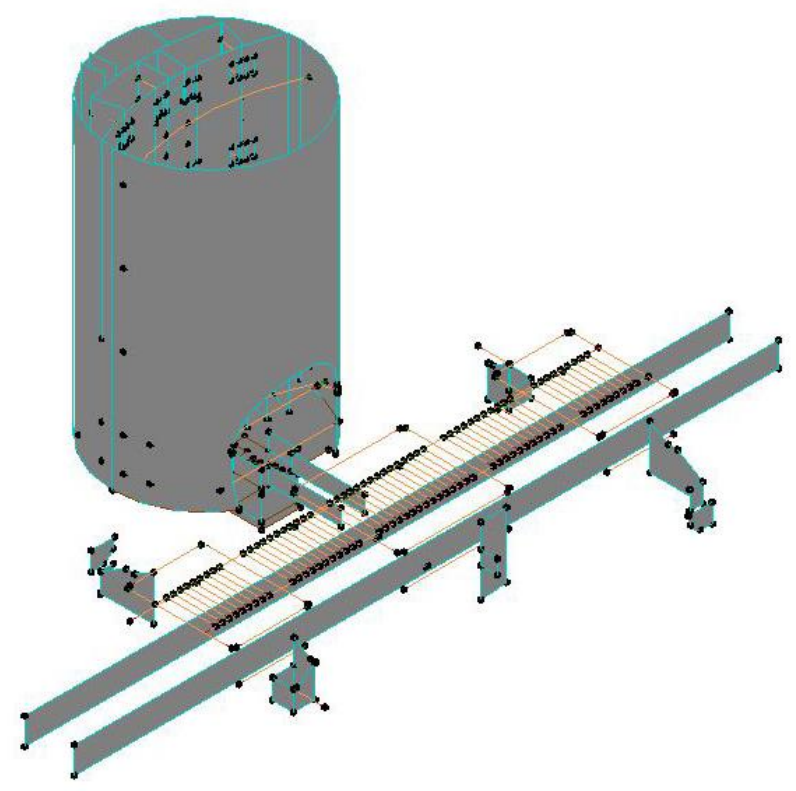

Figura 2: Modelo analítico da estrutura no Revit 2019. 
Nestes casos, o primeiro passo consiste em gerar a geometria simplificada que será utilizada no programa de cálculo. Para tal, recorreu-se ao Dynamo [4]. Foi criada uma rotina que, perante a seleção das faces interior e exterior do elemento, cria uma terceira superfície entre essas duas. O passo seguinte consiste em transportar a referida geometria para o programa de cálculo, neste caso o SAP2000 [4]. Contudo, o único formato em que o Dynamo permite exportar geometrias é o SAT, mas o SAP2000 não permite importações nesse formato. Surgiu então a ideia de utilizar a API do SAP2000 para realizar essa tarefa. A API do SAP2000 é compatível com VBA, VB.NET, C\#, C++, Visual Fortran, Python e Matlab [5]. O Dynamo permite escrever rotinas em IronPython [6]. Foram feitas algumas tentativas para conseguir aceder ao SAP2000 através do Dynamo, mas sem sucesso. Contudo, durante as pesquisas para solucionar o problema surgiu a Geometry Gym.

\section{Geometry Gym}

A Geometry Gym foi fundada em 2009 e nasceu da ideia de gerar modelos de análise estrutural a partir de formas arquitetónicas complexas. Inicialmente entre o Rhinoceros [8] e o GSA, que é o software de análise estrutural da Oasys, que por sua vez é a empresa de software da Arup (empresa multinacional de engenharia e arquitetura). Ao longo do tempo foram desenvolvendo a capacidade de exportar geometrias complexas para outros softwares de análise estrutural. Neste momento, para além do GSA, disponibilizam plug-ins para Etabs, SAP2000, ROBOT, SCIA, Sofistik, SpaceGass e Stand7 [7].

Um dos componentes mais importantes do plug-in é o ggBake, que é responsável por desencadear a exportação do modelo definido no Grasshopper [9] para o software de análise estrutural, abrindo automaticamente a aplicação e gerando o modelo.

A geometria que tinha sido gerada em Dynamo foi então exportada em SAT e importada no Rhinoceros (Figura 3). Utilizaram-se as ferramentas nativas do Grasshopper para gerar a malha de elementos finitos (Figura 4 e Figura 5) e depois o ggBake para transferir o modelo para o SAP2000 (Figura 6).

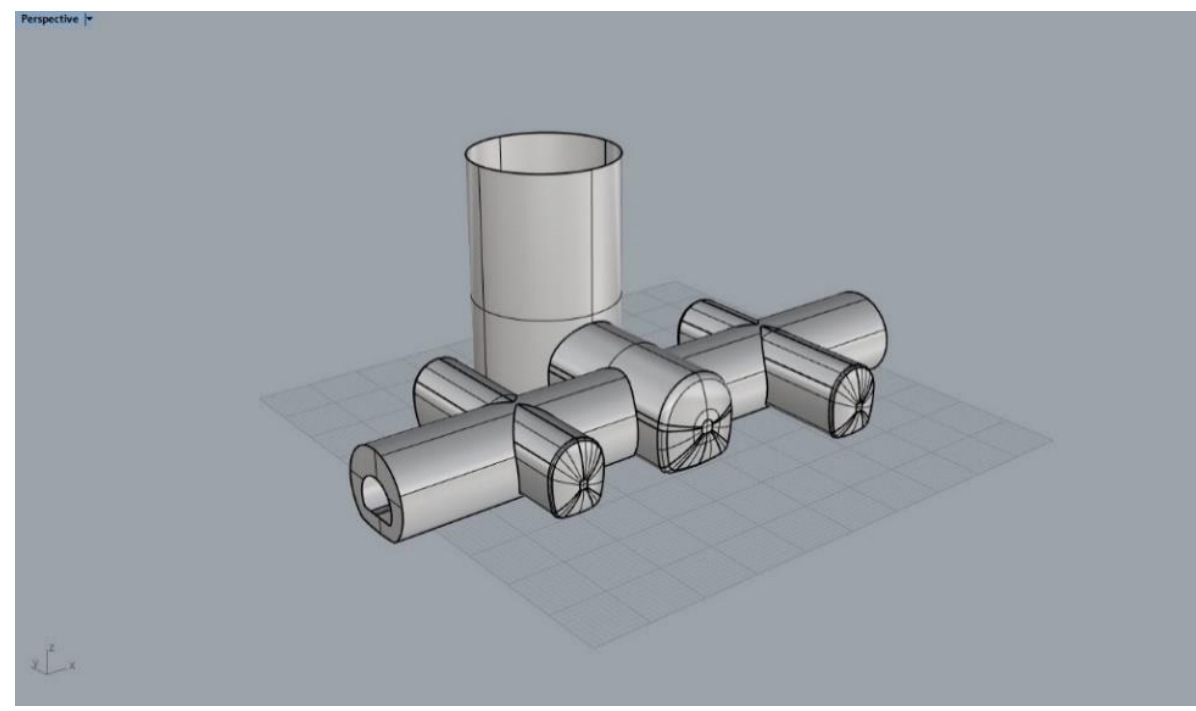

Figura 3: Modelo analítico da estrutura no Rhinoceros 6. 
ptBIM $2020-3^{\circ}$ Congresso Português de Building Information Modelling

26 e 27 de novembro e 4 de dezembro de 2020, Faculdade de Engenharia da Universidade do Porto

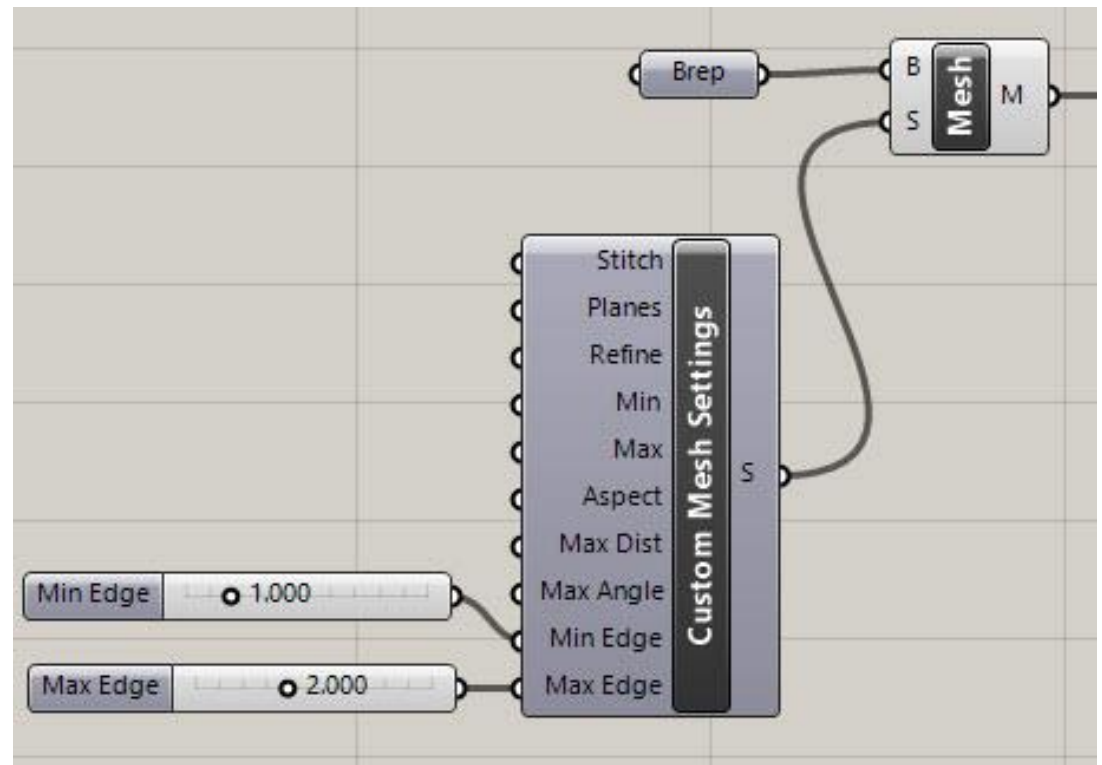

Figura 4: Código que permite gerar a malha de elementos finitos no Grasshopper.

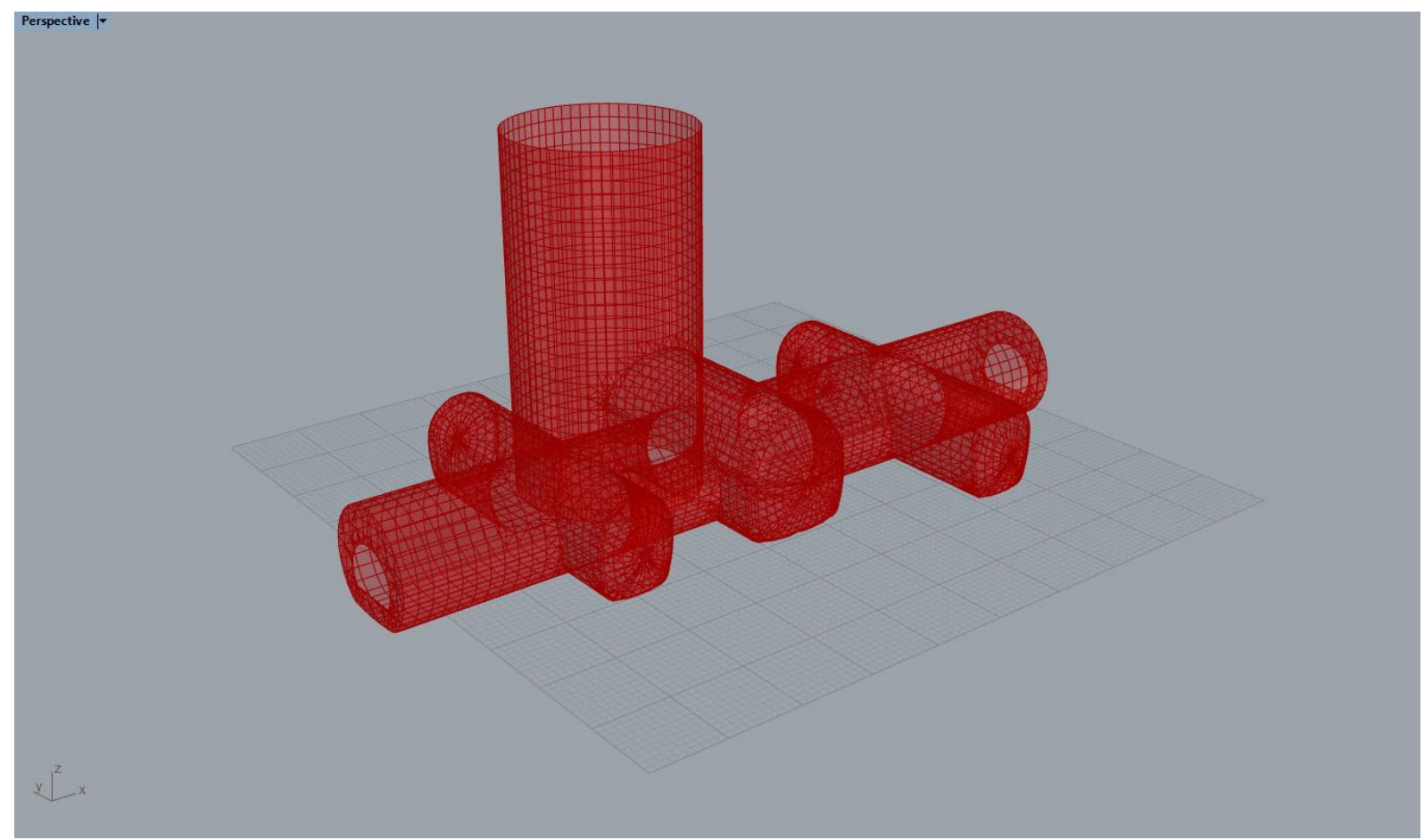

Figura 5: Malha de elementos finitos no Rhinoceros 6. 


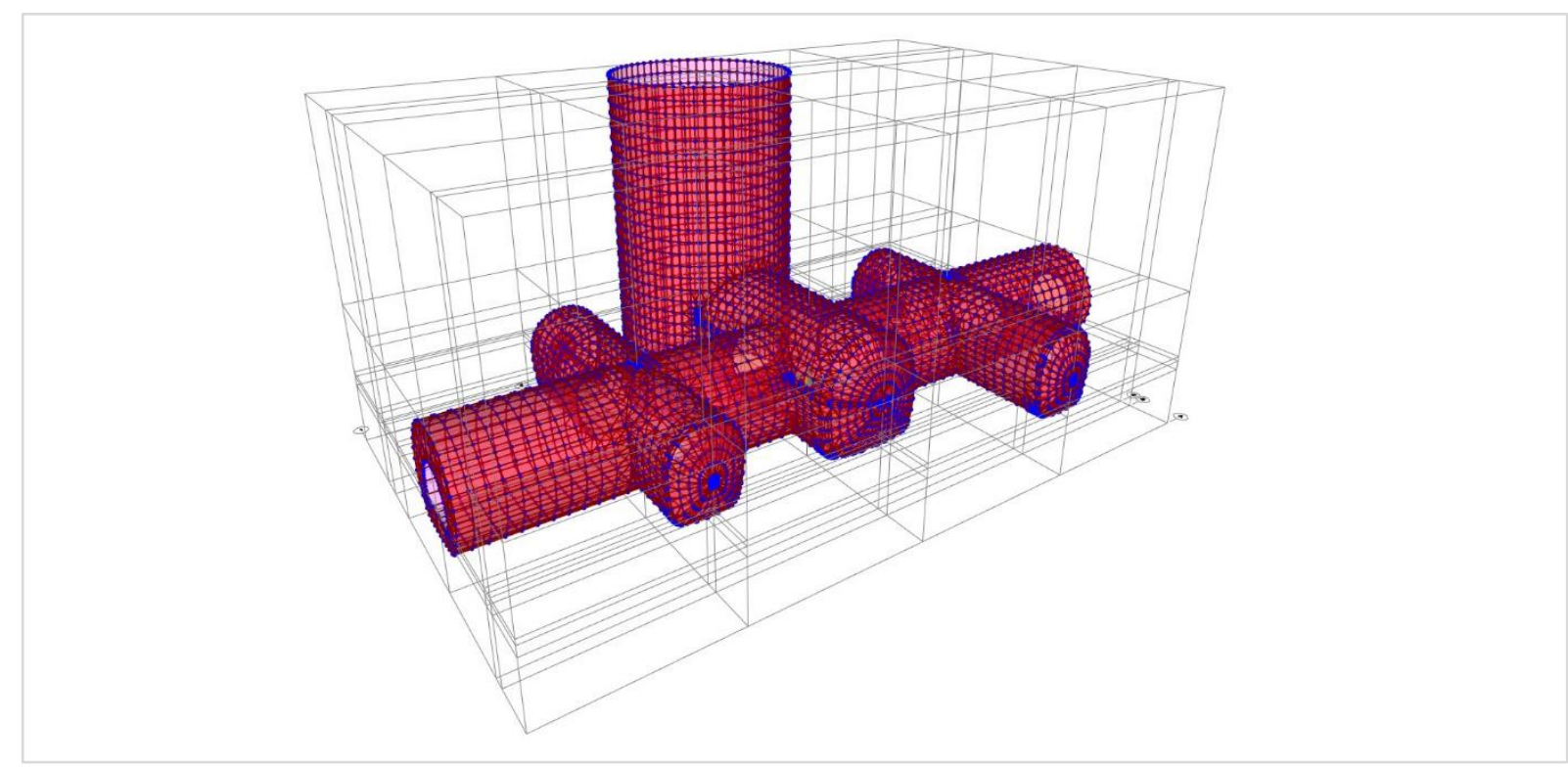

Figura 6: Malha de elementos finitos no SAP2000 v21.

\section{Conclusões}

A relação da engenharia civil com a programação tem oscilado ao longo do tempo, mas a introdução do BIM e dos ambientes de programação visual vieram, sem dúvida, aproximá-las. $\mathrm{Na}$ TPF Consultores criam-se rotinas para pequenas customizações quase diariamente. Em todo o caso, o acompanhamento do mercado é fundamental. Não só para não consumir tempo e recursos a desenvolver rotinas que já estão disponíveis, mas também para se equacionar se é mais vantajoso desenvolver internamente, ou comprar o desenvolvimento de terceiros. No caso concreto da exportação de geometrias complexas para o software de análise estrutural, o recurso ao plug-in da Geometry Gym, foi claramente a melhor opção.

\section{Referências}

[1] B. Raphael e I. Smith, Engineering Informatics. Fundamentals of Computer-Aided Engineering, John Wiley \& Sons, Ltd, 2013.

[2] "Citizen Developer," Gartner, [Online]. Available: https://www.gartner.com/en/information-technology/glossary/citizen-developer. [Acedido em Fevereiro 2020].

[3] "Revit," [Online]. Available: https://www.autodesk.pt/products/revit/overview?plc=RVT\&term=1YEAR\&support=ADVANCED\&quantity=1. [Acedido em Fevereiro 2020].

[4] “Open source graphical programming for design," [Online]. Available: https://dynamobim.org/. [Acedido em Fevereiro 2020].

[5] "SAP2000," [Online]. Available: https://www.csiportugal.com/software/2/sap2000. [Acedido em Fevereiro 2020]. 
[6] “API (Application Programming Interface)," [Online]. Available: https://www.csiportugal.com/estat/40/application-programming-interface-(api)\#. [Acedido em Fevereiro 2020].

[7] "Python," [Online]. Available: https://primer.dynamobim.org/10_Custom-Nodes/104_Python.html. [Acedido em Fevereiro 2020].

[8] "Rhinoceros," [Online]. Available: https://www.rhino3d.com/. [Acedido em Fevereiro 2020].

[9] “About," [Online]. Available: https://geometrygym.wordpress.com/about/. [Acedido em Fevereiro 2020].

[10] “Grasshopper," [Online]. Available: https://www.grasshopper3d.com/. [Acedido em Fevereiro 2020]. 